

\title{
Variabilité génétique de la digestibilité du maïs ensilage mesurée sur des moutons standards
}

\author{
Y. Barrière, J.-C. Émile, R. Traineau, Y. Hébert
}

\section{To cite this version:}

Y. Barrière, J.-C. Émile, R. Traineau, Y. Hébert. Variabilité génétique de la digestibilité du maïs ensilage mesurée sur des moutons standards. Productions Animales, 1992, 5 (4), pp.247-255. hal00895980

\section{HAL Id: hal-00895980 \\ https://hal.science/hal-00895980}

Submitted on 1 Jan 1992

HAL is a multi-disciplinary open access archive for the deposit and dissemination of scientific research documents, whether they are published or not. The documents may come from teaching and research institutions in France or abroad, or from public or private research centers.
L'archive ouverte pluridisciplinaire HAL, est destinée au dépôt et à la diffusion de documents scientifiques de niveau recherche, publiés ou non, émanant des établissements d'enseignement et de recherche français ou étrangers, des laboratoires publics ou privés. 
INRA Prod. Anim.,

1992,5 (4), 247 - 255

\section{Y. BARRIÈRE, J.-C. EMILE, \\ R. TRAINEAU, Y. HÉBERT}

INRA Station d'Amélioration des Plantes Fourragères

86600 Lusignan

\section{Variabilité génétique de la digestibilité du maiis ensilage mesurée sur des moutons standards}

Les rations avec des ensilages de maïs sont à la base de l'alimentation des ruminants à haut potentiel. A partir d'une synthèse de 918 mesures de digestibilité avec des moutons standards, l'importance du choix de l'hybride de maïs sur la digestibilité et la valeur UFL ou UFV de l'ensilage est clairement établi.

Il y a actuellement 3400000 ha de maïs ensilés dans la CEE, dont 1600000 ha en France. Avec l'ensilage de mais, dont la faible teneur en azote est facilement corrigée par des tourteaux, les éleveurs disposent d'une ration de base à forte valeur énergétique, comprise, si l'on se réfère aux tables, entre 0,90 et 0,96 UFL (soit de 0,80 à 0,87 UFV) selon le niveau de maturité et la teneur en grain (Andrieu et

\section{Résumé}

918 mesures de digestibilité d'ensilages de maïs par des moutons standards, qui portaient sur 175 génotypes, ont été récapitulées après des expérimentations réalisées entre 1969 et 1990 à l'INRA de Lusignan. Les analyses de variance montrent que les effets liés au génotype sont toujours très significatifs, même en prenant en compte la précocité des hybrides et en excluant le matériel brown-midrib. Le CUD moyen de la matière organique mesuré $(70,8 \%)$ et les valeurs énergétiques moyennes qui en sont déduites (0,90 UFL et 0,84 UFV) confirment les valeurs données par les tables, mais ces mesures montrent aussi que des différences importantes existent entre génotypes. L'effet du groupe de précocité du matériel génétique est net, les hybrides tardifs ayant une digestibilité de 2,5 points inférieure et une valeur UFL moyenne plus faible de 0,05 UF. Entre hybrides précoces inscrits, les différences sont couramment voisines de 4 points pour le CUD de la matière organique, soit $0,07 \mathrm{UF}$ et peuvent atteindre 8 points, soit 0,15 UF. La valeur énergétique des ensilages n'est pas liée à leur teneur en grain, des ensilages à 0,90 UFL ayant ainsi des teneurs en grain comprises entre 20 et $60 \%$, et réciproquement des ensilages à $48 \%$ de grains ayant des valeurs UFL comprises entre 0,78 et 1,05 . Les corrélations entre productivité et digestibilités ou valeur énergétique sont faibles. Les différences de digestibilité peuvent en partie être reliées aux efforts de sélection pour l'amélioration de la résistance mécanique des plantes à la verse et à la casse. L'intégration d'un critère de sélection prédictif de la digestibilité devrait permettre de maîtriser cette dérive en choisissant les génotypes réalisant un bon compro. mis entre résistance à la verse et valeur énergétique. al 1988). Les valeurs énergétiques données par ces tables ont été établies à partir de mesures des coefficients d'utilisation digestive (CUD) de la matière organique faites avec des moutons maintenus en cage à digestibilité. Ces mesures ont été réalisées avec un nombre limité d'hybrides précoces (Funk245, Dekalb204, Inra258, LG11...), (Andrieu, communication personnelle), dont la base génétique est étroite et qui ne sont pratiquement plus utilisés par les éleveurs, ni représentatifs du matériel génétique effectivement disponible. Actuellement, les variétés de maïs inscrites aux différents catalogues européens sont soit des variétés issues d'une stricte sélection "grain", soit des variétés issues d'une sélection "grain" avec un tri final des hybrides les plus adaptés à une utilisation mixte "grain" et "ensilage", soit enfin des variétés issues d'une sélection pour l'utilisation en ensilage à partir d'un matériel génétique d'autant moins marqué par des décennies de sélection "grain" que l'objectif de recherche des entreprises est plus spécifiquement ciblé sur cet aspect ensilage.

A partir d'une synthèse des données obtenues au cours de 22 années d'expérimentations avec des moutons standards à la station INRA d'amélioration des plantes fourragères de Lusignan (Barrière et al 1992), l'objectif de ce travail était donc d'étudier, chez des hybrides de maiis ensilés, la variabilité génétique de la digestibilité in vivo de la matière organique, de la cellulose, et celle de critères chimiques ou agronomiques auxquels elles pouvaient être reliées, en faisant ici plus particulièrement le lien avec celle de la valeur énergétique, et en comparant les hybrides actuellement disponibles pour les éleveurs, aux anciennes varié- 
tés ayant servi à établir les valeurs UFL ou UFV de référence. En raison du très grand nombre de variétés de mais inscrites en Europe, il n'était pas envisageable de tester l'ensemble des hybrides des catalogues des différents pays. Le choix des hybrides étudiés a été réalisé en tentant de couvrir une gamme de variabilité génétique aussi large que possible, en dépit du caractère confidentiel des formules hybrides. Pour cela, ont été utilisées des variétés provenant de différents obtenteurs, inscrites surtout en France avec ou sans la mention ensilage, mais aussi cultivées ou inscrites en Europe du Nord avec prise en compte ou non de critères de valeur alimentaire. Des hybrides expérimentaux ont élargi cette base génétique avec l'utilisation de lignées qui ne sont pas présentes dans les hybrides inscrits. Des hybrides demi-tardifs et tardifs ont aussi été inclus, en particulier parce que les mesures de référence avaient été réalisées uniquement avec des hybrides précoces. Enfin, des hybrides à nervures brunes (brown-midrib) ont permis d'ajouter une source particulière de variation, les gènes récessifs bm1 ou bm3 modifiant le métabolisme des phénols, conduisant à une diminution de la teneur en lignine, une modification de la composition chimique de la lignine, avec une augmentation de la digestibilité de la cellulose et à une amélioration des performances zootechniques (Rook et al 1977, Sommerfeldt et al 1979, Hoden et al 1985, Malterre et al 1985)

\section{1 / Principales conditions expérimentales}

Les expérimentations ont été réalisées entre 1969 et 1990 à Lusignan (Vienne) dans un milieu qui se caractérise souvent par des printemps frais suivis d'étés chauds et relativement secs, avec une irrigation d'appoint à partir de 1975. Les maïs ont été cultivés à une densité qui variait de 75 à 95000 plantes par hectare en fonction de la précocité des hybrides. L'unité de culture pour une mesure était constituée de deux (quelquefois une) parcelles de $150 \mathrm{~m}^{2}$ ensilées en mini-silos expérimentaux (Traineau 1991) à un stade de maturité tel que les teneurs en matière sèche des ensilages soient comprises entre 30 et $35 \%$, valeurs qui n'étaient parfois pas atteintes pour certains hybrides tardifs, ou au contraire dépassées par certains hybrides très précoces. La teneur en azote de la ration était équilibrée par addition d'urée. Les pertes de produits volatils durant l'étuvage ont été estimées à partir des méthodes proposées par Dulphy et al (1975 et 1981).

Les mesures de valeur alimentaire ont été réalisées dans les conditions standards sur des moutons Texel en cages à digestibilité, alimentés ad libitum. Elles ont porté sur les quantités ingérées et les CUD de la matière organique et de la cellulose brute. Les valeurs UFL et UFV ont été calculées à partir des régressions établies par Andrieu et Demarquilly
(1987) ; les valeurs UFV données sont à diminuer de $5 \%$ pour prendre en compte les interactions digestives négatives entre fourrage et concentrés chez les bovins à l'engrais (Andrieu et al 1988). Bien évidemment, il y a par construction une très forte liaison entre ces valeurs énergétiques et le CUD de la matière organique, ce qui se retrouve au niveau des corrélations (tableau 3). La teneur en cellulose brute, la teneur en matières azotées et les quantités ingérées entrent également dans les régressions, avec des pondérations plus ou moins importantes. Des mesures de digestibilité in vitro ont été réalisées avec la méthode enzymatique APC de Lila et al (1986) sur la plupart des hybrides étudiés à partir de 1981 (solubilisations successives de l'échantillon par une amylase, une pepsine et un mélange standard de cellulases et d'hémicellulases).

918 mesures de digestibilités ont ainsi été récapitulées, correspondant à l'étude de 175 hybrides dont 90 variétés inscrites au catalogue français ou à un catalogue européen, 67 hybrides expérimentaux et 18 hybrides à nervures brunes ( $15 \mathrm{bm} 3$ et $3 \mathrm{bm} 1$ ). Ces hybrides se répartissaient dans les 6 groupes de précocité du catalogue français. Dans les discussions ultérieures, les hybrides très précoces, précoces et demi-précoces cornés*dentés ont été rassemblés sous le terme "précoces", les autres étant dénommés "tardifs". Dans les tableaux de résultats, les hybrides inscrits récemment ont été codés $\mathrm{Rh}$ pour des raisons de confidentialité ; les hybrides expérimentaux ont été codés Ex.

Les analyses de variance ont été réalisées selon des modèles classiques à effets fixes en prenant en compte un effet année, un effet silo dans année, un effet génotype hiérarchisé ou non au groupe de précocité, et en excluant ou non les génotypes brown-midrib (Barrière et al 1992 ; logiciels INRA Amance et Modli). Outre l'observation répétée de certains génotypes plusieurs années, 4 hybrides régulièrement étudiés (LG11, Inra260, Inra258 et Dea) ont réalisé la connection statistique entre années et permis les estimations de moyennes.

\section{2 / Résultats}

\section{1 / Une importante variabilité liée à l'hybride de maïs}

Les moyennes, écarts-types et valeurs des $\mathrm{F}$ de Fisher pour les principaux caractères étudiés sont donnés dans le tableau 1. Les effets liés aux génotypes sont toujours significatifs, même quand l'effet précocité est pris en compte. La variation entre génotypes pour la teneur en cellulose brute est apparue relativement faible, alors que la variabilité de sa digestibilité était très élevée. L'écart-type génotypique du CUD de la cellulose brute est ainsi plus de deux fois plus élevé que celui du CUD de la matière organique, lié à une dilution de la cellulose au sein de composés dont la digestibilité 


\begin{tabular}{|l|c|c|c|c|}
\hline & Moyenne & Ecart-type & F génotype & $\begin{array}{c}\text { F génotype } \\
\text { /précocité }\end{array}$ \\
\hline Teneur en matière sèche (\%) & 34,4 & 5,6 & 4,5 & 3,2 \\
Teneur en grain (\%) & 41,4 & 7,6 & 5,9 & 4,7 \\
Teneur en cellulose brute (\%) & 19,5 & 2,7 & 3,7 & 2,8 \\
Rendement (t/ha) & 12,9 & 2,8 & 4,1 & 3,1 \\
Quantités ingérées (g/kg 0,75) & 49,9 & 7,1 & 1,8 & 1,7 \\
CUD matière organique (\%) & 70,8 & 3,7 & 6,9 & 4,1 \\
CUD cellulose brute (\%) & 55,2 & 8,6 & 3,2 & 2,8 \\
UFL & 0,90 & 0,06 & 6,8 & 3,4 \\
UFV & 0,84 & 0,07 & 6,9 & 3,4 \\
\hline
\end{tabular}

Tableau 1. Valeurs moyennes, écart-types et $F$ de Fisher des principaux caractères mesurés sur les 918 micro-silos étudiés. ( $F$ significatifs au seuil de 1\%)

Figure 1. Histogramme de fréquence des différents hybrides dans chacune des classes de valeur UFL entre 0,75 et 1,00 . est élevée (amidon, glucides solubles, protéines, lipides ...). Le critère digestibilité de la cellulose semble donc plus important que le critère quantité de cellulose tant pour le sélectionneur que pour l'utilisateur de maïs ensilage. Les valeurs moyennes obtenues ici pour le CUD de la matière organique et les valeurs énergétiques UFL et UFV correspondent exactement à celles données par les tables pour un ensilage de maîs ayant subi des conditions de végétation normales. En revanche, le CUD de la cellulose brute des tables est supérieur de 5 points à la valeur obtenue ici pour nos ensilages.

Les histogrammes de fréquence des hybrides dans chacune des classes de valeurs UFL entre 0,75 et 1,00 sont rassemblés dans la figure 1. Les valeurs moyennes et intervalles de confiance pour les CUD de la matière organique et les valeurs UFL et UFV de quelques hybrides inscrits, expérimentaux ou brownmidrib sont données à titre d'illustration dans le tableau 2. L'observation de l'ensemble des données met tout d'abord en évidence une grande variabilité entre hybrides pour la digestibilité de la matière organique et la valeur énergétique, avec un effet moyen net du niveau de précocité. Les hybrides tardifs ont ainsi un CUD de la matière organique et une valeur énergétique moyenne respectivement inférieures de 2,5 points et 0,05 UFL à ceux des hybrides précoces (brown-midrib exclus). Cela est autant lié à des qualités de la partie non-grain de l'ensilage différentes, avec ainsi 3,0 points d'écarts entre les CUD de la cellulose brute des précoces et des tardifs, qu'à un effet de teneurs en grain, en moyenne plus faibles de 2,7 points chez les tardifs. Les écarts maximum observés entre hybrides inscrits de précocité comparable sont en général voisins de 4 et 17 points pour respectivement les CUD de la matière organique et de la cellulose brute; mais ils peuvent atteindre respectivement 8 et 23 points. En terme de valeur énergétique, cela conduit à des écarts courants de 0,07 UFL, qui peuvent atteindre 0,15 UFL entre des variétés précoces. $57 \%$ des hybrides précoces inscrits étudiés ont une digestibilité de la matière organique comprise entre 69 et $72 \%$, $15 \%$ sont en dessous de cet intervalle et $28 \%$ au dessus. En terme de valeur énergétique $61 \%$ des hybrides se situent entre 0,87 et 0,92 UFL, $16 \%$ se situent en-dessous et $23 \%$ audessus. Les hybrides inscrits récemment sont présents dans toutes les classes de digestibilité


64 génotypes précoces inscrits

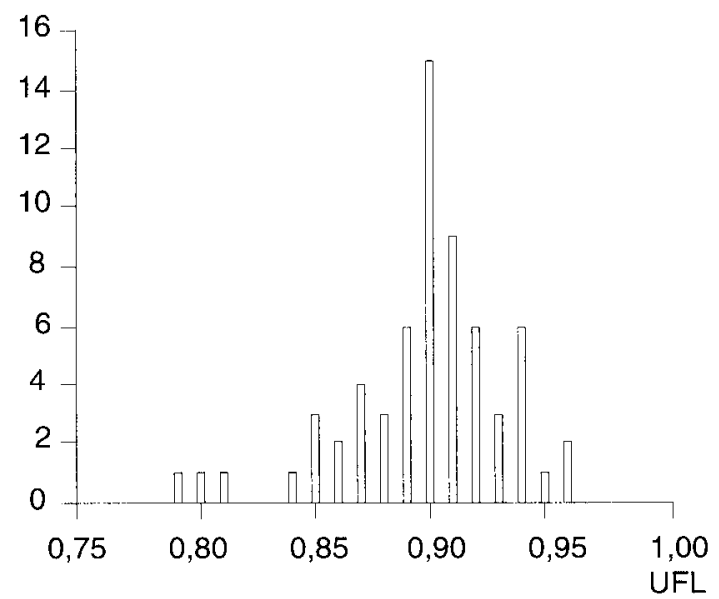


Tableau 2. Moyennes estimées des CUD de la matière organique, des valeurs énergétiques UFL et UFV, et intervalles de confiance (ic), d'hybrides inscrits ou expérimentaux. (Les valeurs UFV doivent être diminuées de 5\% conformément aux recommandations des tables)

\begin{tabular}{|c|c|c|c|c|c|c|c|c|}
\hline HYBRIDES & $\begin{array}{c}\text { Année } \\
\text { d'inscription }\end{array}$ & $\begin{array}{l}\text { nombre } \\
\text { de silos }\end{array}$ & CUD MO & ic & UFL & ic & UFV & ic \\
\hline \multicolumn{9}{|l|}{$\begin{array}{l}\text { Hybrides } \\
\text { "PRÉCOCES" }\end{array}$} \\
\hline très précoces & & & & & & & & \\
\hline Rh151 & 1988 & 2 & 70,1 & 2,6 & 0,89 & 0,05 & 0,83 & 0,05 \\
\hline Brulouis & 1980 & 10 & 71,8 & 1,2 & 0,92 & 0,02 & 0,86 & 0,02 \\
\hline Browning & 1985 & 2 & 73,1 & 2,6 & 0,94 & 0,05 & 0,89 & 0,05 \\
\hline Rh174 & 1991 & 2 & 74,1 & 2,6 & 0,95 & 0,05 & 0,90 & 0,06 \\
\hline Puma & 1982 & 2 & 73,8 & 2,6 & 0,96 & 0,05 & 0,91 & 0,06 \\
\hline Rh169 & 1987 & 2 & 74,2 & 2,6 & 0,96 & 0,05 & 0,91 & 0,06 \\
\hline Puma bm $_{3}$ & - & 2 & 74,9 & 2,6 & 0,97 & 0,05 & 0,92 & 0,06 \\
\hline \multicolumn{9}{|l|}{ précoces } \\
\hline Rh84 & 1977 & 2 & 66,6 & 2,6 & 0,81 & 0,05 & 0,74 & 0,05 \\
\hline Ascot & 1988 & 6 & 69,8 & 1,6 & 0,87 & 0,03 & 0,81 & 0,03 \\
\hline Brutus & 1978 & 4 & 71,1 & 2,0 & 0,89 & 0,03 & 0,83 & 0,04 \\
\hline LG11 & 1970 & 72 & 71,1 & 0,5 & 0,90 & 0,01 & 0,84 & 0,01 \\
\hline Dekalb250 & 1986 & 9 & 71,3 & 1,3 & 0,90 & 0,02 & 0,84 & 0,03 \\
\hline Inra258 & 1958 & 36 & 72,9 & 0,7 & 0,93 & 0,01 & 0,88 & 0,01 \\
\hline Lixis & 1985 & 14 & 72,8 & 1,1 & 0,93 & 0,02 & 0,87 & 0,02 \\
\hline $\mathrm{LG} 11 \mathrm{bm}_{1}$ & - & 18 & 72,5 & 0,9 & 0,93 & 0,02 & 0,87 & 0,02 \\
\hline Funk245 & 1970 & 17 & 73,2 & 1,0 & 0,94 & 0,02 & 0,89 & 0,02 \\
\hline Rh168 & 1988 & 2 & 72,7 & 2,6 & 0,94 & 0,05 & 0,89 & 0,06 \\
\hline Rh131 & 1986 & 2 & 73,8 & 2,6 & 0,94 & 0,05 & 0,89 & 0,05 \\
\hline LG11 bm 3 & - & 26 & 74,2 & 0,7 & 0,95 & 0,01 & 0,90 & 0,02 \\
\hline Inra258 $\mathrm{bm}_{3}$ & - & 4 & 74,1 & 1,8 & 0,96 & 0,03 & 0,91 & 0,04 \\
\hline Ex35 & - & 1 & 74,5 & 3,6 & 0,97 & 0,06 & 0,92 & 0,07 \\
\hline \multicolumn{9}{|l|}{ 1/2 précoces } \\
\hline Rh162 & 1990 & 2 & 65,1 & 2,6 & 0,79 & 0,05 & 0,71 & 0,06 \\
\hline Rh161 & 1989 & 4 & 65,7 & 2,0 & 0,80 & 0,04 & 0,72 & 0,04 \\
\hline Mammouth & 1982 & 6 & 68,8 & 1,5 & 0,86 & 0,03 & 0,79 & 0,03 \\
\hline Helga & 1989 & 4 & 69,8 & 2,8 & 0,87 & 0,04 & 0,81 & 0,04 \\
\hline Adonis & 1984 & 2 & 71,3 & 2,6 & 0,89 & 0,05 & 0,83 & 0,06 \\
\hline Inra260 & 1961 & 51 & 71,0 & 0,6 & 0,90 & 0,01 & 0,84 & 0,01 \\
\hline Dea & 1980 & 32 & 71,7 & 0,7 & 0,91 & 0,01 & 0,86 & 0,01 \\
\hline Rh129 & 1985 & 2 & 72,3 & 2,6 & 0,92 & 0,05 & 0,86 & 0,05 \\
\hline Inra260 bm 1 & - & 13 & 72,0 & 1,0 & 0,92 & 0,02 & 0,86 & 0,02 \\
\hline Rh167 & 1987 & 2 & 73,5 & 2,6 & 0,94 & 0,05 & 0,88 & 0,06 \\
\hline Inra $260 \mathrm{bm}_{3}$ & - & 27 & 73,4 & 0,8 & 0,94 & 0,01 & 0,89 & 0,02 \\
\hline Ex64 & - & 2 & 76,0 & 2,7 & 0,99 & 0,05 & 0,95 & 0,06 \\
\hline \multicolumn{9}{|l|}{$\begin{array}{l}\text { Hybrides } \\
\text { "TARDIFS" }\end{array}$} \\
\hline Lorena & 1981 & 8 & 65,0 & 1,4 & 0,79 & 0,02 & 0,71 & 0,03 \\
\hline Roc & 1977 & 4 & 66,0 & 1,9 & 0,80 & 0,03 & 0,73 & 0,04 \\
\hline Rh153 & 1986 & 2 & 66,6 & 2,6 & 0,83 & 0,05 & 0,76 & 0,06 \\
\hline Rh152 & 1988 & 2 & 66,9 & 2,6 & 0,83 & 0,05 & 0,76 & 0,06 \\
\hline Inra508 & 1969 & 6 & 68,9 & 1,5 & 0,87 & 0,03 & 0,80 & 0,03 \\
\hline Iowa 4417 & 1951 & 10 & 69,9 & 1,3 & 0,88 & 0,02 & 0,81 & 0,03 \\
\hline Rh154 & 1988 & 2 & 69,4 & 2,6 & 0,88 & 0,05 & 0,82 & 0,06 \\
\hline Brio & 1974 & 2 & 71,8 & 2,6 & 0,91 & 0,05 & 0,85 & 0,05 \\
\hline
\end{tabular}




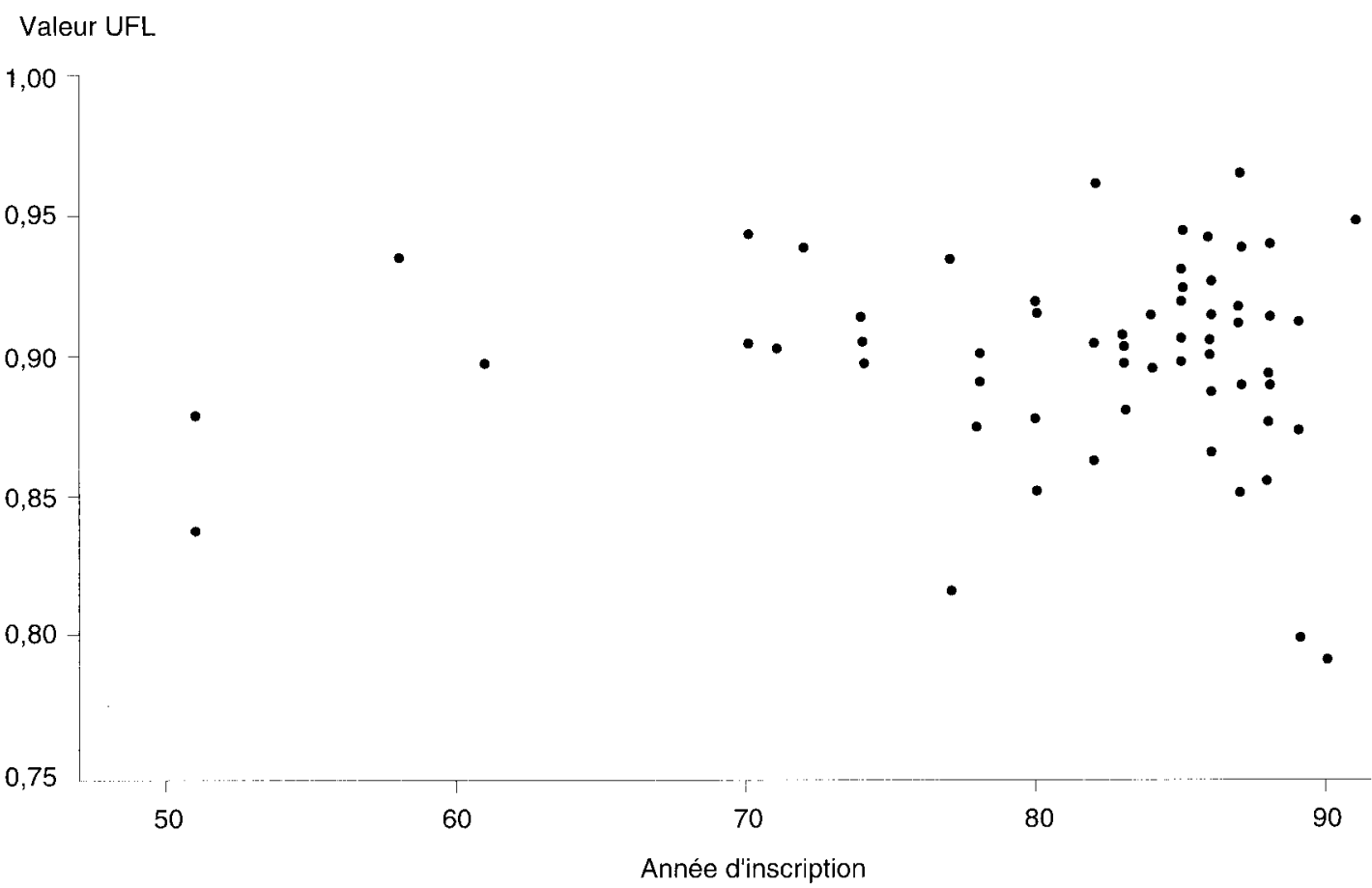

de la matière organique ou de valeur énergétique (figure 2), certains d'entre eux ayant toutefois une valeur énergétique particulièrement faible. Les valeurs énergétiques des anciens témoins (LG11, Inra258, Funk245) sont légèrement supérieures à la moyenne, avec 0,92 UFL. Les bonnes ou moins bonnes digestibilités ou valeurs énergétiques de certains hybrides confirment la connaissance que l'on pouvait avoir de ces génotypes, avec, par exemple, la plutôt faible valeur de Ascot et Helga, et au contraire la bonne valeur de Lixis. Toutefois, la valeur obtenue pour Brutus est plutôt inférieure à ce que l'on pouvait attendre après les mesures in vitro de digestibilité de parois faites aux Pays-Bas sur cet hybride.

\section{2 / Des critères classiques peu liés à la valeur énergétique}

Les corrélations phénotypiques entre les CUD de la matière organique, les valeurs énergétiques UFL ou UFV et les principaux caractères mesurés sont données dans le tableau 3 ; l'étude des corrélations génétiques a été présentée par Barrière et al (1992). Comme cela avait déjà été observé avec des récoltes à différents stades de maturité (Andrieu et Demarquilly 1974, Barrière et al 1991), il n'y a pas d'effets de la teneur en matière sèche sur la valeur énergétique du maîs ensilage (à partir de $25 \%$ ); il y a en revanche un effet bien connu sur les quantités ingérées.

La liaison entre les teneurs en grain et le CUD de la matière organique ou la valeur énergétique apparaît ici particulièrement faible, surtout pour les hybrides précoces, avec des corrélations proches de zéro. Contrairement à une idée largement répandue, ce n'est pas, ou pas seulement, sa teneur en grain qui fait la bonne ou moins bonne valeur énergétique d'un ensilage de maïs. La figure 3 montre ainsi que la valeur énergétique d'un ensilage de maïs à $50 \%$ de grain varie entre 0,80 et $1,05 \mathrm{UFL}$, et que, réciproquement, des ensilages de mais à 0,90 UFL ont une teneur en grain comprise entre 20 et près de $60 \%$. Des ensilages de mais relativement pauvres en grain (35-40\%) ont une valeur énergétique qui varie entre 0,70 et $1,05 \mathrm{UFL}$, avec une moyenne de 0,88 UFL (ou 0,82 UFV). En revanche, les ensilages ayant une teneur en grain supérieure ou égale respectivement à 45 et $50 \%$ ont une valeur énergétique moyenne qui n'atteint respectivement que 0,92 et $0,93 \mathrm{UFL}(0,86$ et $0,87 \mathrm{UFV})$, alors que la valeur donnée dans les tables pour des ensilages très riches en grain est de 0,96 UFL (0,91 UFV).

Parmi les caractères étudiés, la teneur en cellulose brute et le CUD de cette même cellulose, sont ceux qui sont le mieux liés au CUD de la matière organique, et de façon relativement indépendante puisque la liaison entre ces deux caractéristiques est relativement faible, avec une corrélation de $-0,36$ (en se limitant aux hybrides précoces). Le CUD de la cellulose brute explique près des deux tiers de la variation observée pour le CUD de la matière organique et un peu plus de la moitié de la variation pour la valeur énergétique ; la teneur en cellulose brute explique moins de la moitié de la variation observée pour la digestibilité et la valeur énergétique, en dépit de la prise en compte de ce facteur dans les régressions. Les liaisons négatives entre rendement et digestibilité ou valeurs UF sont également assez faibles, permettant de choisir des hybrides à la fois productifs et à bonnes digestibilités ou valeurs énergétiques. Par ailleurs, les liaisons entre quantités ingérées et digestibilités ou
Figure 2. Relation entre valeur UFL et année d'inscription (64 hybrides précoces).

\section{La valeur énergétique de l'ensilage semble peu liée à sa teneur en grain.}


Figure 3. Relation entre valeur UFL et teneur en grains (918 mesures)

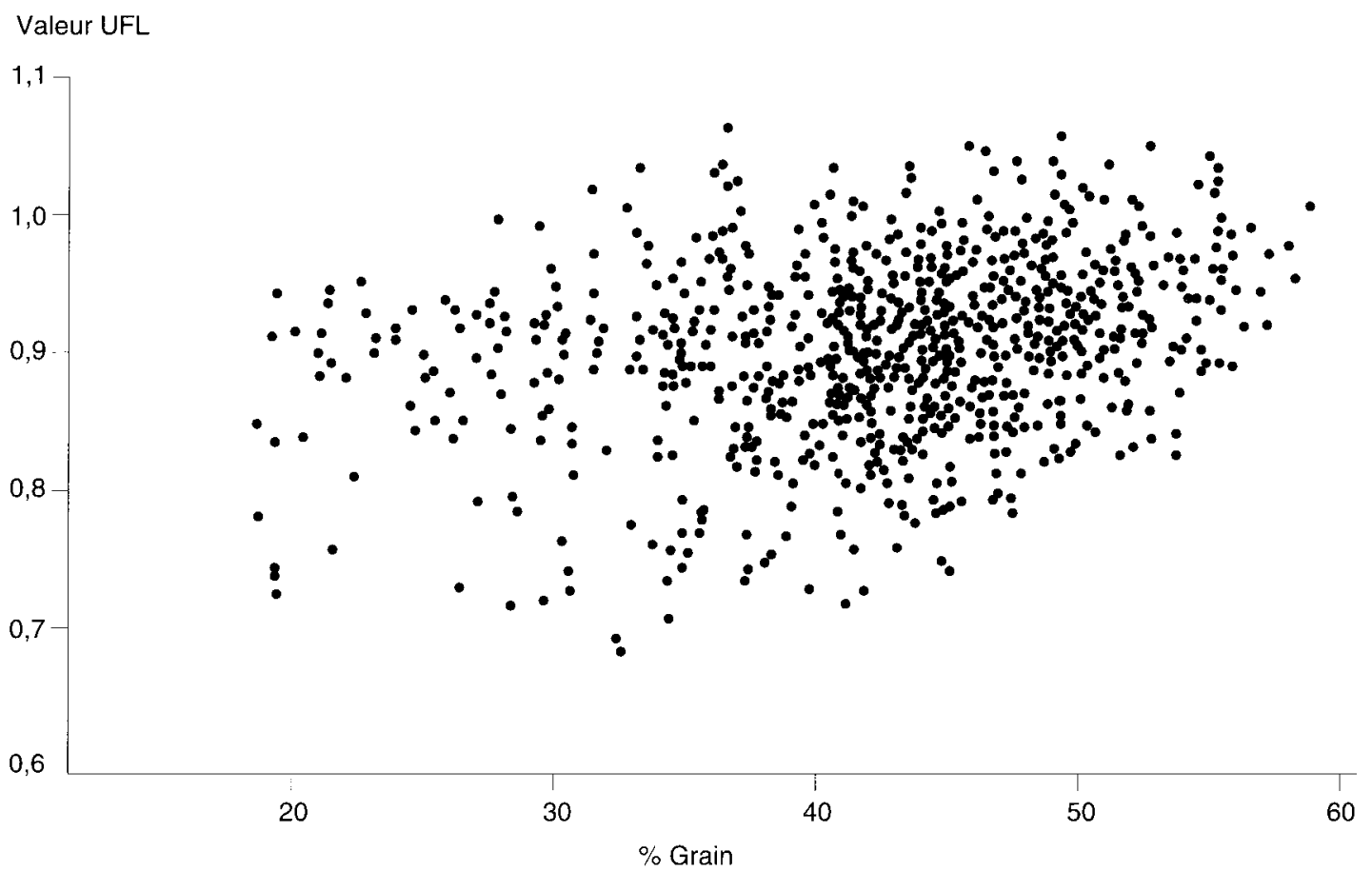

Tableau 3. Corrélations phénotypiques entre CUD de la matière organique, valeurs énergétiques et caractères étudiés sur l'ensemble des observations (918 sauf $\left(^{*}\right) 436$ observations), et en se limitant aux génotypes précoces (hybrides tardifs ou brown-midrib exclus) (581 sauf ( $\left.{ }^{*}\right) 321$ observations).

\begin{tabular}{|l|rrr|rrr|}
\hline & \multicolumn{3}{|c|}{ Ensemble des génotypes } & \multicolumn{3}{c|}{ Génotypes précoces } \\
\hline & CUD MO & UFL & UFV & CUD MO & UFL & UFV \\
\hline & & & & & & \\
CUD MO & - & 0,98 & 0,98 & - & 0,97 & 0,97 \\
Teneur en M. sèche & $-0,01$ & 0,01 & 0,05 & $-0,17$ & $-0,17$ & $-0,18$ \\
Teneur en grain & 0,18 & 0,27 & 0,26 & 0,04 & 0,13 & 0,12 \\
Teneur en cellulose & $-0,72$ & $-0,68$ & $-0,69$ & $-0,72$ & $-0,66$ & $-0,67$ \\
CUD cellulose & 0,80 & 0,73 & 0,73 & 0,74 & 0,66 & 0,66 \\
Quantités ingérées & $-0,14$ & $-0,04$ & $-0,04$ & $-0,18$ & $-0,03$ & $-0,04$ \\
Rendement & $-0,51$ & $-0,43$ & $-0,43$ & $-0,45$ & $-0,34$ & $-0,35$ \\
Digestibilité APC ${ }^{*}$ * & 0,46 & 0,39 & 0,40 & 0,50 & 0,42 & 0,44 \\
& & & & & & \\
\hline
\end{tabular}

même valeurs UF sont pratiquement nulles (tableau 3). Au niveau intra-spécifique maïs avec des moutons standards, les quantités ingérées seraient donc sans effet sur la valeur énergétique. Sachant par ailleurs qu'il semble exclu de prédire des quantités ingérées autrement qu'au sein d'une espèce animale donnée et à un stade physiologique donné (Chesnot et Martin-Rosset 1985, Moran et al 1988), l'intérêt de cette mesure de quantité ingérée par les moutons standards apparait donc limité dans le cas du maïs ensilage. En raison des relativement faibles liaisons entre les valeurs UF et les teneurs en grain ou en cellulose brute, il n'est sans doute pas raisonnable de calculer à partir de ces données des équations de prédiction des valeurs énergétiques, qui seraient alors peu performantes.

\section{3 / Discussion}

\section{1 / Des conséquences pour les valeurs énergétiques pour des gros ruminants}

Ces résultats ont été obtenus avec des moutons standards alimentés à des niveaux voisins des besoins d'entretien, et il convient de s'interroger pour savoir dans quelle mesure ils sont transposables aux vaches laitières ou aux bovins en croissance. Tout d'abord, il faut rappeler que, d'une part ces conditions de mesures sur moutons standards ont été établies pour prédire la valeur énergétique de référence des aliments consommés par des gros ruminants, et que, d'autre part, il y a, par construction, une très forte liaison entre la digestibilité de la 
matière organique mesurée par les moutons standards et la valeur énergétique. Un certain nombre de travaux avec différents végétaux ont d'ailleurs montré que les classements de digestibilité de la matière organique étaient semblables pour les moutons et les bovins, avec toutefois une digestibilité supérieure de 2 à $6 \%$ chez les bovins (Chenost et MartinRosset 1985, Aerts et al 1986, Moran et al 1988). Une expérimentation réalisée sur vaches laitières à Lusignan, avec des ensilages de mais, a toutefois mis en évidence une valorisation énergétique supérieure à la valeur UFL estimée à partir de la digestibilité mesurée sur des moutons pour un hybride dont la digestibilité de la cellulose était élevée (Barrière et Emile 1990). Ce résultat devrait être confirmé par d'autres mesures sur maïs, mais il est en accord avec les observations de Chenost et Martin-Rosset (1985) qui avaient obtenu, avec des fourrages pauvres en amidon, des digestibilités de la cellulose brute supérieures de $9 \%$ en moyenne chez les bovins comparés aux ovins. Pour ce qui est des effets de la teneur en grain pour des vaches laitières, la réduction de la teneur en grain d'ensilages de maïs de 48 à $41 \%$ ne modifie ni les productions laitières, ni la qualité du lait produit, mais conduit au contraire à une meilleure valorisation énergétique des ensilages appauvris en grain (Emile et Barrière 1992). Des comparaisons d'ensilage de variétés de maïs dont les CUD et valeurs UF sont nettement différents sont en cours sur vaches laitières et taurillons. Ils permettront de préciser des écarts éventuels de comportement entre gros bovins et moutons, et de mesurer de quelle façon des écarts de 0,15 UFL obtenus à partir de mesures de digestibilité sur moutons sont transposables aux gros ruminants. En attendant, il est sans doute raisonnable de considérer que les différentes valeurs UFL obtenues avec les moutons sont de bons prédicteurs des valeurs de valorisation par les gros bovins. Les corrections éventuelles pourraient porter sur une possible meilleure valorisation des celluloses de bonne qualité, au moins par les vaches laitières. Dans ces conditions, et en fonction des écarts mis en évidence entre variétés de maïs, des différences de 0,07 ou 0,15 UFL correspondent respectivement à des différences d'apports journaliers de 1,05 et 2,25 UFL pour une vache laitière ingérant journellement 15 $\mathrm{kg}$ de MS d'ensilage de maïs, soit l'énergie nécessaire à la production de 2,4 et $5,1 \mathrm{~kg}$ de lait standard à apporter, ou non, sous forme de concentrés de production.

\section{2 / De possibles liaisons négatives entre digestibilités et tenues de plantes}

La variabilité génotypique observée entre hybrides peut d'abord être reliée au fond génétique de l'hybride en général, certaines lignées conduisant en moyenne à des hybrides à forte ou faible digestibilité. En l'absence de sélection pour la valeur alimentaire, ces différences pourraient être soit aléatoires, soit résulter d'une dérive vers un type génétique ou un autre sous la pression de sélection pour les autres caractères agronomiques. Les corrélations génétiques entre la productivité en biomasse et les CUD de la matière organique ou de la cellulose brute étant pratiquement nulles (Barrière et al 1992), les différences entre hybrides peuvent s'expliquer en partie par les efforts de sélection plus ou moins intenses réalisés sur des caractéristiques liées à la tenue des plantes. La résistance à la verse en végétation, qualité indispensable aux maïs cultivés pour le grain ou l'ensilage, est liée à un bon développement racinaire et à une bonne résistance mécanique des racines à l'arrachement. Objectif de sélection important surtout pour des variétés utilisées comme maïs grain, la résistance à la verse ou à la casse à maturité et à surmaturité se fonde sur deux caractéristiques, d'une part le maintien de la turgescence des tissus au cours de la maturation, et d'autre part une meilleure résistance mécanique de la tige. Si les aspects de croissance racinaire sont probablement indépendants des facteurs liés à la digestibilité des parois et si un meilleur maintien de l'aspect vert est sans doute favorable, en revanche une meilleure résistance mécanique des tissus peut conduire à une plus faible digestibilité des parois de la plante. On peut sans doute ainsi relier la faible digestibilité de certains hybrides au progrès génétique net pour la résistance à la verse à maturité enregistré au cours des dernières décennies (Derieux et al 1987). Des hybrides de bonne digestibilité comme Inra258 ont aussi de faibles qualités de tiges, alors que des hybrides comme Rh161 et Rh162 sont au contraire résistants aux verses. Mais ces liaisons ne sont pas absolues, et il sera possible de progresser à la fois en qualité de tiges et en valeur alimentaire, à condition de disposer d'un test pour prédire les digestibilités sur un grand nombre d'échantillons, et sans doute d'accepter une résistance mécanique des plantes relativement moindre ou différente. D'un point de vue biochimique, des différences de teneurs respectives en acides phénoliques monomères de la lignine ont été mises en évidence entre hybrides normaux et bm3, qui devront être confirmées entre hybrides normaux différant pour la digestibilité de la cellulose.

\section{3 / Des possibilités de prédiction en routine de la digestibilité et de la valeur $\mathrm{UF}$}

Les corrélations entre teneur en grain et valeur énergétique sont insuffisantes pour prédire de façon satisfaisante cette valeur énergétique. En revanche, la liaison apparait plus élevée, et négative, entre la teneur en cellulose brute et la valeur énergétique (tableau 3). Par ailleurs, à condition d'estimer les moyennes génotypiques au travers d'un dispositif expérimental comprenant des mesures répétées (figure 4), les valeurs APC permettent au moins de trier des génotypes à digestibilité ou valeur UF plus élevées, et d'éliminer des géno-

\section{La faible digestibilité de certains hybrides peut être liée à une meilleure résistance à la verse à maturité, critère important de sélection pour les variétés de maïs grain.}


Figure 4. Relation entre valeur UFL et digestibilité in vitro par la méthode APC (moyennes estimées pour 112 génotypes).

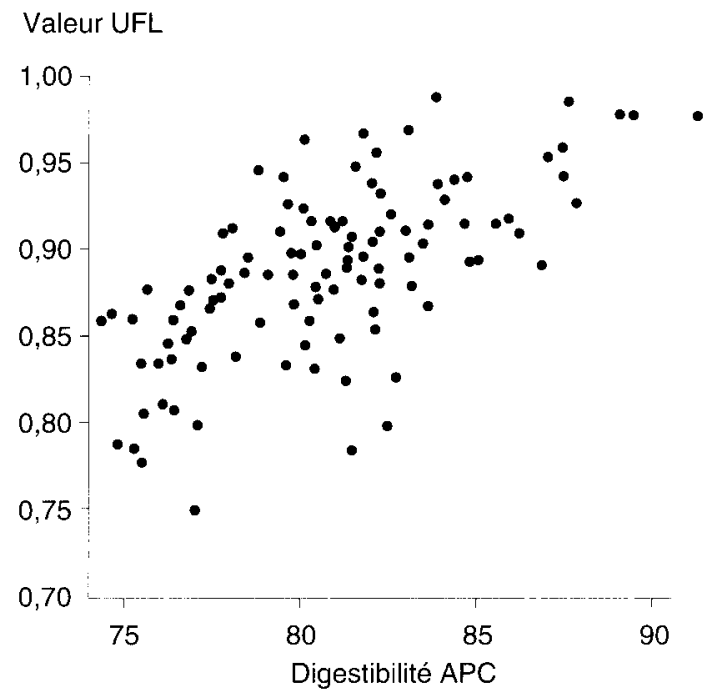

types à faibles valeurs. De plus, les résultats du "Club digestibilité" (Dardenne et al 1992) ont montré que la prédiction des valeurs de CUD de la matière organique, et donc les valeurs UF, pouvait être encore améliorée en utilisant une régression multicaractère qui ajoute à une mesure de solubilité enzymatique telle que LG-Ensitec (Ronsin et Femenias 1990) différents critères de composition chimique (cellulose brute, matières azotées ...), conduisant à une corrélation voisine de 0,7 entre valeurs prédites et valeurs mesurées. Avec ce type d'équation, il est possible d'envisager une prédiction en routine des valeurs de CUD ou des valeurs UF des ensilages de maïs, d'autant que les paramètres entrant dans les régressions peuvent être prédits dans de très bonnes conditions en spectrométrie de réflectance dans le proche infra-rouge (NIRS). La principale limite à l'utilisation de ces équations, outre leur relative imprécision, est la qualité de l'échantillonnage dans les remorques ou les silos, les résultats les plus satisfaisants étant obtenus dans des dispositifs structurés permettant une interprétation statistique des données, ce qui est le cas au cours des programmes de sélection. Les résultats du "Club digestibilité" ont aussi montré qu'il était possible de prédire directement les valeurs de CUD de la matière organique et les valeurs UF par NIRS. Mais l'utilisation en routine de cette méthode suppose d'abord l'obtention de références supplémentaires avec des moutons standards afin d'assurer la pertinence de l'équation de calibration, puis, pendant un certain nombre d'années au moins, la réalisation régulière de mesures sur animaux pour pren-dre en compte de façon convenable les effets années. La prédiction du CUD de la cellulose brute pourrait être réalisée simultanément. Dans ce cas également, la qualité des résultats sera à la mesure de la qualité de l'échantillonnage et de celle du dispositif statistique.

\section{Conclusion}

Pour un éleveur, différentes conclusions découlent de ces essais. Tout d'abord, le choix de la variété de maïs est un élément important à prendre en compte dans la mise en oeuvre d'une ration à base de maïs ensilage. Mesurée sur les moutons dans les conditions de référence, des différences notables de CUD et de valeur énergétique entre variétés ont été mises en évidence, qui peuvent avoir une incidence importante sur la rentabilité économique de l'élevage. Le choix de génotypes à bonne valeur énergétique doit ainsi permettre de calculer les rations sur la base de 0,95 UFL $(0,86$ UFV) pour des maiis en conditions de végétation normales au lieu de la valeur 0,90 UFL habituellement utilisée (0,80 UFV). Réciproquement, ce choix variétal doit permettre d'éviter des variétés dont la valeur alimentaire se situe très probablement au-dessous des valeurs énergétiques de référence. Il faut noter, d'autre part, la faible importance des teneurs en grain, un hybride plus pauvre en grain pouvant avoir une valeur énergétique plus élevée que celle d'un hybride riche. Cet aspect est particulièrement intéressant à prendre en compte au niveau d'une ration où la complémentation conduit à augmenter encore la teneur en amidon, contribuant donc à augmenter les interactions digestives négatives au niveau du rumen (E1 Shazy et al 1961, Faverdin et al 1987). Dans un contexte général de politique agricole restrictive sur les prix et sur les quantités des productions animales, il ne faudra en aucune manière négliger des possibilités d'améliorer les marges des exploitations par ce simple choix de l'hybride de maïs cultivé, conduisant à une économie de concentrés pour un niveau constant de production animale.

\section{Remerciements}

Nous remercions l'ensemble des personnes qui ont participé directement ou par leurs conseils à ce travail, en particulier L. Huguet, A. Gallais, G. Bertin, M. Lila, V. Furstoss, C. Demarquilly, J. Andrieu, M. Largeaud, S. Allerit, M. Bucher, J.L. Doux, C. Pradeau et J. Moinet.

\section{Références bibliographiques}

Aerts J.V., 1986. Use of sheep as pilot animals for predicting digestibility of feeds to be fed to dairy cows. IDF Bulletin 196 : $22-24$.

Andrieu J., Demarquilly C., 1974. Valeur alimentaire du mais fourrage. II. Influence du stade de végétation, de la variété, du peuplement, de l'enrichissement en épis et de l'addition d'urée sur la digestibilité et l'ingestibilité de l'ensilage de maïs. Ann. Zootech., $23: 1-25$.

Andrieu J., Demarquilly C., 1987. Valeur nutritive des fourrages : tables et prévision. Bull. Techn. CRZV Theix, INRA, 70 : $61-73$

Andrieu J., Demarquilly C., Sauvant D., 1988. Tables de la valeur nutritive des aliments. In Alimentation des bovins, ovins et caprins, sous la direction de R. Jarrige, INRA éditeur pages 351-464. 
Barrière Y., Emile J.C., 1990. Effet des teneurs en grain et de la variabilité génétique sur la valeur énergétique du mais ensilage mesurée par des vaches laitières. Agronomie $10: 201-221$.

Barrière Y., Demarquilly C., Hebert Y., Dardenne P., Andrieu J., Maupetit P., Lila M., Emile J.C., 1991. Influence de la variabilité génétique et environnementale sur la digestibilité in vitro ou in vivo du mais fourrage. Agronomie $3: 151-157$.

Barrière Y., Traineau R., Emile J.C., Hébert Y., 1992. Variation and covariation of silage maize digestibility estimated from digestion trials with sheep. Euphytica, $59: 61-72$.

Chenost M., Martin-Rosset W., 1985. Comparaison entre espèces (mouton, cheval, bovin) de la digestibilité et des quantités ingérées des fourrages verts. Ann. Zootech., 34 : 291-312.

Dardenne P., Andrieu J., Barrière Y., Biston R., Demarquilly C., Femenias N., Lila M., Maupetit P., Rivière F., Ronsin T., 1992. Composition and nutritive value of whole maize plants fed fresh to sheep. II Prediction of the in vivo organic matter digestibility. Soumis pour publication aux Annales de Zootechnie.

Derieux M., Darrigrand M., Gallais A., Barrière Y., Bloc D., Montalant Y., 1987. Estimation du progrès génétique réalisé chez le maïs grain en France entre 1950 et 1985. Agronomie 7 : $1-11$.

Dulphy J.P., Demarquilly C., Henry M., 1975. Perte de composés volatils lors de la détermination à l'étuve de la teneur en matière sèche des ensilage. Ann. Zootech. 24 : 743-756.

Dulphy J.P., Demarquilly C., 1981. Problèmes particuliers aux ensilages. In Prévision de la valeur nutritive des aliments des ruminants. INRA éditeur, 81-104.

El-Shazy K., Dehority B.A., Johnson R.R., 1961. Effect of starch on the digestion of cellulose in vitro and in vivo by rumen microorganisms. J. anim. Sci. $20: 268-273$.

Emile J.C., Barrière Y., 1992. Effets de la teneur en grain de l'ensilage de maïs sur les performances zootechniques de vaches laitières. INRA, Prod. anim. 5 : 113-120.
Faverdin P., Hoden A., Coulon J.B., 1987. Recommandations alimentaires pour les vaches laitières. Bull Tech C.R.Z.V. Theix, INRA, $70: 132-152$

Hoden A., Barrière Y., Gallais A., Huguet L., Journet M., Mourguet A. 1985. Le mais brown-midrib plante entière. III Utilisation sous forme d'ensilage par des vaches laitières. Bull. techn. CRZV Theix, INRA $60: 43-58$

Lila M., Barrière Y., Traineau R., 1986. Mise au point et étude d'un test enzymatique de la digestibilité de fourrages pauvres ou riches en amidon. Agronomie $6: 285-291$.

Malterre C. Bertin G., Gallais A., Huguet L., Micol D. 1985. Le maïs brown-midrib plante entière. III Utilisation sous forme d'ensilage par des taurillons. Bull. techn. CRZV Theix, INRA 60 $43-58$

Moran J.B., Lemerle C., Trigg T.E., 1988. The intake and digestion of maize silage-based diet by dairy cows and sheep. Anim Feed Sci and Technol. 20, 239-312.

Ronsin T., Femenias N., 1990. Use of NIRS determination of quality in a silage maize breeding program. 3rd international Near Infrared Spectroscopy Conference, Bruxelles, Belgique; 2529 Juin 1990

Rook J.A., Muller L.D., Shank D.B. 1977. Intake and digestibility of brown midrib corn silage by lactating dairy cows. J. Dairy Sci. 60 : 1894-1904.

Sommerfeldt J.L., Schingoethe D.J., Muller L.D., 1979. Brown midrib corn silage for lactating dairy cows. J. Dairy Sci. 62 : $1611-1618$

Traineau R., 1991. Mise au point d'un matériel de récolte et de confections de silos adapté à des parcelles expérimentales. Fourrages, 128, 465-470.

\section{Summary}

Genetic variation of in vivo digestibility and energetic feed value of maize silage.

Variability for in vivo digestibility of organic matter (DOM) and energetic feed values UFL and UFV of maize silage , and their relationships with agronomic and others digestibility traits were reported from a compilation of 918 experiments with standard sheep. Genotype effects of DOM and UFL values were highly significant, even if the earliness of the hybrids was taken into account and brown-midrib hybrids were excluded from the study. Observed means for DOM $(70.8 \%)$, UFL and UFV values ( 0.90 UFL and 0.84 UFV) were similar to usual table values. The DOM of late genotypes were 2.5 points lower than that of earlier hybrids, corresponding to 0.05 UFL for energetic values. Differences between early hybrids were often about 4 points for DOM and could reach 8 points, corresponding respectively to 0.07 and 0.15 UFL. Phenotypic correlation between UFL values and grain contents was very low, but higher and negative with cellulose content. Yield was not clearly related to DOM or UFL values within each group of earliness, allowing quality improvement without agronomic drift. There was no obvious correlation between year of registration of hybrids and DOM or UFL values, but the worst values were often observed for newer hybrids. IVDOM, according to the APC process, was not a very good predictor of UFL values. But such enzymatic process could probably be used to avoid drift towards poor UFL values with hybrids bred for higher stalk strength. For a stock-breeder, a pertinent choice of a maize hybrid might allow significant increase in concentrates and money saving.

Y. BARRIERE, J.-C. ÉMILE, R.TRAINEAU, Y. HÉBERT, 1992. Variabilité génétique de la digestibilité du maiss ensilage mesurée sur des moutons standards. INRA Prod. Anim., 5 (4) 247-255. 